\title{
Fatores associados e predição da retinopatia da prematuridade
}

\author{
Retinopathy of prematurity: associated factors and \\ prediction
}

\author{
Rafaela Pereira ${ }^{1}$, Rosângela Santana Getirana ${ }^{1}$, Taqueco Teruya Uchimura ${ }^{1}$, \\ Darci Aparecida Martins Corrêa ${ }^{2}$, Isolde Previdelli ${ }^{1}$, Emerson Barili ${ }^{1}$
}

\begin{abstract}
Resumo
Introdução: A retinopatia da prematuridade (ROP) é uma doença vasoproliferativa, que afeta a retina das crianças que nascem prematuramente; assim, este estudo propôs identificar fatores associados e predizer a retinopatia na prematuridade. Método: Realizou-se um estudo transversal, com recém-nascidos prematuros, dividindo-os em dois grupos: com e sem retinopatia. As variáveis foram categorizadas por meio do teste da razão de verossimilhança e as variáveis que obtiveram $p$-valores menores que 0,2 foram analisadas por um modelo logístico múltiplo. A capacidade de predição do modelo foi realizada por meio da construção da curva ROC. Resultados: Dos 296 recém-nascidos, 32 (10,81\%) apresentaram retinopatia. No modelo logístico, as variáveis que apresentaram fator de risco foram idade gestacional $\leq 30$ semanas ( $p$-valor=0,0214), peso ao nascer $\leq 1.250$ gramas $(p$-valor=0,0013) e escore Apgar no primeiro minuto $<7$ ( $p$-valor=0,0352). A curva ROC com sensibilidade 0,771 e especificidade 0,774 evidenciou o modelo com precisão de 0,8448 e ponto de probabilidade de 0,79. Conclusões: Este modelo pode ser usado para predição de retinopatia em recém-nascidos prematuros com um fator de risco de 0,79 , destacando que estes necessitam de maior atenção. Palavras-chave: retinopatia da prematuridade; fatores de risco; modelos logísticos.
\end{abstract}

\begin{abstract}
Introduction: Retinopathy of prematurity (ROP) is a vasoproliferative disease that affects the retina of prematurely born children. Therefore, this study proposed to identify associated factors and to predict retinopathy in prematurity. Method: A cross-sectional study including preterm newborns divided into two groups: with and without retinopathy. The variables were categorized using the likelihood ratio test, and the variables with p-values less than 0.2 were analyzed by a multiple logistic model. The model used the construction of a Receiver Operating Characteristic (ROC) curve as predicting tool. Results: Of the 296 newborns, 32 (10.81\%) had retinopathy. The following variables represented a risk factor according to the logistic model: gestational age $\leq 30$ weeks $(p$-value $=0.0214)$, birth weight $\leq 1250$ grams $(p$-value $=0.0013)$ and Apgar score at 1 minute $<7(p$-value $=0.0352)$. The ROC curve with sensitivity of 0.771 and specificity of 0.774 was a precise model, showing accuracy of 0.8448 and a probability point of 0.79. Conclusions: This model can be used to predict retinopathy in premature newborns with a risk factor of 0.79 , emphasizing that the need for additional attention.
\end{abstract}

Keywords: retinopathy of prematurity; risk factors; logistic models.

'Departamento de Estatística (DES), Universidade Estadual de Maringá (UEM) - Maringá (PR), Brasil.

2 Departamento de Enfermagem (DEN), Universidade Estadual de Maringá (UEM) - Maringá (PR), Brasil.

Trabalho realizado no Hospital Universitário, Universidade Estadual de Maringá (UEM) - Maringá (PR), Brasil.

Endereço para correspondência: Emerson Barili - Departamento de Estatística (DES), Universidade Estadual de Maringá (UEM), Av. Colombo, 5790 - Jardim Universitário - CEP: 87020-900 - Maringá (PR), Brasil - Email: ebarili2@gmail.com

Fonte de financiamento: Pesquisa com apoio financeiro da Fundação Araucária Chamada Pública 09/2015 - Programa Bolsa Sênior. Protocolo 45.767 Área Ciências Exatas e da Terra.

Conflito de interesses: nada a declarar. 


\section{INTRODUÇÃO}

A retinopatia da prematuridade (ROP) é uma doença vasoproliferativa, que afeta a retina das crianças que nascem prematuramente. O desenvolvimento da doença poderá levar à destruição da estrutura do globo ocular e, consequentemente, à cegueira total e irreversível ${ }^{1,2}$.

A retinopatia da prematuridade se apresenta como a segunda maior causa de cegueira infantil no Brasil e uma das principais causas no mundo ${ }^{3}$. Estima-se que, no Brasil, a cada ano, cerca de 15 mil crianças nascidas pré-termo estão expostas ao risco de desenvolver a ROP na sua forma mais grave e que 500 novos casos de cegueira pela ROP ocorram a cada ano ${ }^{3}$.

Os fatores de risco mais consistentes para o surgimento da ROP são o peso ao nascer $\leq 1.500$ gramas, a idade gestacional $\leq 32$ semanas e a oxigenoterapia em alta concentração por longo período de tempo. Entre os fatores menos consistentes, estão a falta de vitamina $\mathrm{E}$, a exposição à luz intensa e a sepse ${ }^{2}$.

O Conselho Brasileiro de Oftalmologia, a Sociedade Brasileira de Oftalmologia Pediátrica e a Sociedade Brasileira de Pediatria preconizam o exame oftalmológico de fundo de olho para recém-nascidos prematuros com peso $\leq 1.500$ gramas e idade gestacional $\leq 32$ semanas, e, segundo as diretrizes publicadas em 2007, para todos os recém-nascidos com mais de 1.500 gramas e/ou que desenvolveram outras comorbidades relacionadas com o nascimento prematuro, tais como a Síndrome do Desconforto Respiratório, a sepse, as transfusões sanguíneas, a gestação múltipla e a hemorragia intraventricular ${ }^{4}$. Acrescenta-se ainda, que o primeiro exame oftalmológico de fundo de olho deve ser realizado entre a quarta e a sexta semana de vida, e os exames subsequentes, a cada uma ou duas semanas, conforme o diagnóstico do exame anterior ${ }^{5-7}$. O rastreamento é feito pela oftalmoscopia indireta, por um profissional devidamente habilitado, geralmente o médico.

Muitos estudos consideram o ponto de corte para o risco da ROP o peso ao nascer $\leq 1.500$ gramas ou a idade gestacional $\leq 32$ semanas; outros consideram que, com este ponto de corte, ocorrem muitos falsos negativos, ou seja, a criança deixa de ser de risco e não recebe a devida assistência. Assim, neste estudo, utilizaram-se os dados dos recém-nascidos admitidos no Programa Atenção Humanizada ao Recém-Nascido de Baixo Peso - Método Canguru, que foi implantado em 2004, considerando-se os riscos a que as crianças prematuras estavam expostas ${ }^{8}$. O presente estudo se propôs a verificar a prevalência, propor pontos de corte para as variáveis da ROP, identificar os fatores de risco associados e predizer o diagnóstico da ROP na criança.

\section{MÉTODO}

Foi realizado um estudo transversal retrospectivo no período de janeiro de 2006 a novembro de 2015, com recém-nascidos prematuros admitidos no Programa Atenção Humanizada ao Recém-Nascido de Baixo Peso - Método Canguru, do Hospital
Universitário de Maringá-PR, que possuía o registro do exame de fundo de olho. Os dados foram coletados das fichas do respectivo programa, que foram preenchidas pelos profissionais da saúde, e, quando necessário, dos prontuários das crianças.

Todos os recém-nascidos deste estudo fizeram o teste de triagem do reflexo vermelho ou o teste de fundo de olho. Os critérios para a classificação da retinopatia da prematuridade foram os propostos por The International Classification of Retinopathy of Prematurity Revisited ${ }^{9}$, definindo a doença, de acordo com a gravidade, em cinco estágios. A avaliação oftalmológica consistiu na oftalmoscopia indireta sob dilatação pupilar utilizando-se colírios de tropicamida $1 \%$ ou ciclopentolato $1 \%$ e fenilefrina $2,5 \%$. Uma gota de cada com intervalo de 5 minutos em cada olho, e 40 minutos antes do exame ${ }^{10}$.

O diagnóstico da ROP, quando presente, foi classificado de acordo com a gravidade da alteração causada na retina (estágios da ROP), para assim verificar a prevalência em cada estágio. Para a variável resposta, foi utilizado o diagnóstico da ROP em duas categorias, classificadas como presente ou ausente no recém-nascido prematuro. Para a análise dos fatores associados à ROP, foram analisadas as seguintes variáveis: i) idade da mãe; ii) estado civil; iii) pré-natal; iv) sexo do recém-nascido; v) idade gestacional; vi) tipo de parto; vii) uso de oxigenoterapia; viii) intercorrências na gestação; ix) peso ao nascer; $x$ ) escore Apgar no primeiro minuto; xi) escore Apgar no quinto minuto, e xii) dias totais de internação (DTI).

A análise univariada foi apresentada através da média \pm desvio padrão, bem como a odds ratio bruta, com intervalo de confiança de $95 \%$ e respectivo p-valor. As variáveis quantitativas significantes foram categorizadas de acordo com os pontos de corte apropriados, por meio do teste da razão de verossimilhança. A categorização foi realizada com o intuito de conseguir um ponto de corte de risco para as variáveis associadas à ROP e pela facilidade na interpretação da odds ratio, optando-se pelas categorias, consideradas, na literatura, como categorias de menor risco para serem as baselines.

As variáveis que apresentaram um $\mathrm{p}$-valor menor que 0,2 , na análise univariada ${ }^{11}$, foram consideradas na regressão logística múltipla. A seleção das variáveis foi realizada pelo teste da razão de verossimilhança, em nível de significância de 0,05. Para verificar o poder de predição do modelo, construíram-se as curvas ROC (Receiving Operator Caracteristics) e TG-ROC (Two-Graph Receiving Operator Caracteristics), sendo observada a área sob a curva $R O C$ e um ponto de probabilidade predito, para discriminar os recém-nascidos com ROP e os recém-nascidos sem a ROP. As análises estatísticas foram realizadas no programa SAS 9.4 pelas procedures GENMOD e LOGISTIC ${ }^{12}$.

Para cumprir a Resolução 466/2012 do Conselho Nacional de Saúde, o presente estudo foi aprovado pelo Comitê Permanente de Ética em Pesquisa Envolvendo Seres Humanos (COPEP) da 
Universidade, sob o Parecer n. ${ }^{\circ}$ 060/2015 e com autorização do Hospital Universitário. O Termo de Consentimento Livre e Esclarecido (TCLE) não foi utilizado visto que os dados eram secundários.

\section{RESULTADOS}

No período de janeiro de 2006 a novembro de 2015, foram admitidas no Programa Atenção Humanizada ao Recém-Nascido de Baixo Peso - Método Canguru do Hospital Universitário, 316 crianças; destas, foram excluídas: uma criança que evoluiu a óbito nas primeiras semanas de vida, duas crianças que foram transferidas para outros hospitais da região e 17 que não possuíam o registro do exame do fundo do olho, totalizando então 296 crianças. Das crianças inclusas no estudo, 144 (48,65\%) eram do sexo feminino e $152(51,35 \%)$ do sexo masculino. O peso médio ao nascer foi de $1.662,54 \pm 505,11 \mathrm{~g}$ e a média da idade gestacional foi de 32,08 \pm 2,76 semanas.

A prevalência da ROP em seus vários estadiamentos evolutivos foi de 10,81\% (32 crianças), sendo que 13 (40,63\%) apresentaram ROP no estágio 1; 10 (31,25\%) com ROP no estágio 2; 8 (25\%) com ROP no estágio 3, e apenas $1(3,12 \%)$ criança apresentou ROP no estágio 5.

Para a população do estudo, 149 (50,33\%) crianças utilizaram a oxigenoterapia e 122 (41,22\%) mães apresentaram intercorrências durante a gestação, tais como a doença hipertensiva específica da gravidez, os sangramentos, o descolamento precoce da placenta, a Síndrome de Hellp, a embolia pulmonar, entre outras.
Ressalta-se que as variáveis oxigenoterapia e intercorrências apresentaram grande quantidade de dados faltantes, 120 (40,54\%) e 134 (45,27\%), respectivamente. E, desta forma, não foram selecionadas no estudo.

Na Tabela 1, é apresentada a associação entre as variáveis preditoras e ROP. As variáveis idade gestacional, peso ao nascer, escore Apgar no primeiro e quinto minuto e dias totais de internação apresentaram significância estatística, e foram categorizadas (Tabela 2).

As variáveis categorizadas que apresentaram $\mathrm{p}$-valor $<0,2$ na análise univariada entraram no modelo logístico múltiplo e, após a seleção de variáveis, o modelo resultante ficou com três fatores, excluindo escore de Apgar no quinto minuto e dias totais de internação. Pela Tabela 3, observa-se que as categorias idade gestacional $\leq 30$ semanas, peso ao nascer $\leq 1.250$ gramas e escore de Apgar no primeiro minuto $<7$ se apresentaram como categorias de risco para a ROP.

$\mathrm{Na}$ Figura 1, a área sob a curva $R O C$ apresentou um valor 0,8489; este número representa uma discriminação excelente, segundo Hosmer e Lemeshow ${ }^{11}$, do modelo entre quem tem ROP e quem não tem ROP. O ponto em que a sensibilidade e a especificidade são ótimas, observado pelo gráfico da TG-ROC, resulta na sensibilidade de 0,771 e uma especificidade de 0,774 , conseguindo classificar corretamente 24 de 32 crianças com ROP e 205 de 264 crianças sem ROP. Este ponto de probabilidade foi de 0,79 , o que indica que, se calculada a probabilidade através do modelo logístico para um dado recém-nascido, com suas

Tabela 1. Distribuição das variáveis maternas, de parto e neonatais, segundo a ocorrência de ROP

\begin{tabular}{cccccc} 
Variáveis & ROP presente & ROP ausente & p-valor & OR & IC (95\%) \\
Peso (g) & $1161,093 \pm 41,18$ & $1723,32 \pm 488,01$ & $<, 0001$ & 0,996 & $0,995-0,998$ \\
IG (sem) & $28,90 \pm 2,38$ & $32,46 \pm 2,55$ & $<, 0001$ & 0,614 & $0,519-0,725$ \\
Apgar $1^{\circ}$ min & $4,83 \pm 2,73$ & $6,77 \pm 2,3$ & $<, 0001$ & 0,751 & $0,651-0,865$ \\
Apgar 5 $5^{\circ}$ min & $7,19 \pm 2,53$ & $8,57 \pm 1,63$ & 0,0001 & 0,734 & $0,624-0,864$ \\
Idade da mãe & $25,75 \pm 4,64$ & $26,0 \pm 6,98$ & 0,8408 & 0,994 & $0,941-1,05$ \\
DTI & $64.73 \pm 30.53$ & $31,38 \pm 21,18$ & $<, 0001$ & 1,048 & $1,031-1,064$ \\
Sexo Fem. & $15(46,87 \%)$ & $129(48,86 \%)$ & 0,832 & 0,923 & $0,443-1,925$ \\
Fez PN & $28(93,33 \%)$ & $245(96,07 \%)$ & 0,484 & 0,571 & $0,119-2,74$ \\
Parto Normal & $8(27,58 \%)$ & $85(34,41 \%)$ & 0,463 & 0,726 & $0,308-1,708$ \\
Mãe não casada & $13(46,42 \%)$ & $108(47,16 \%)$ & 0,932 & 0,949 & $0,290-3,101$ \\
\hline
\end{tabular}

ROP = retinopatia da prematuridade; IG = idade gestacional; DTI = dias totais de internação; PN = Pré-Natal ; OR = ODDS RATIO; IC(95\%) = Intervalo de Confiança

Tabela 2. Análise bivariada entre ROP e demais variáveis

\begin{tabular}{cccccc} 
Variáveis & ROP presente & ROP ausente & p-valor & OR & IC (95\%) \\
IG $\leq 30($ sem) & 25 & 55 & $<, 0001$ & 13,571 & $5,77-33,02$ \\
Peso $\leq 1.250(\mathrm{~g})$ & 23 & 41 & $<.0001$ & $<, 02$ & $6,00-32,18$ \\
Apgar $1^{\circ}<7$ & 23 & 84 & $<, 0001$ & 5,81 & $2,5-13,55$ \\
Apgar $5^{\circ}<7$ & 8 & 22 & 0,0056 & 3,65 & $1,46-9,12$ \\
$40<$ DTI $\leq 60$ (dias) & 8 & 40 & 0,0016 & 6,00 & $1,97-18,25$ \\
DTI $>60$ & 16 & 23 & $<, 0001$ & 20,86 & $7,42-58,67$ \\
\hline
\end{tabular}

ROP = retinopatia da prematuridade; IG = idade gestacional; DTI = dias totais de internação; OR = ODDS RATIO; IC(95\%) = Intervalo de Confiança 
Tabela 3. Modelo logístico múltiplo para a Retinopatia da prematuridade (ROP) em recém-nascidos do Programa Atenção Humanizada ao Recém-Nascido de Baixo Peso - Método Canguru

\begin{tabular}{ccccc} 
Variáveis & Estimativa \pm Erro padrão & p-valor & OR & IC (95\%) \\
Intercepto & $3,97 \pm 0,47$ & $<0,0001$ & & \\
IG $\leq 30$ semanas & $1,30 \pm 0,56$ & 0,0214 & 3,67 & $1,21-11,12$ \\
Peso $\leq 1.250$ gramas & $1,66 \pm 0,52$ & 0,0013 & 5,29 & $1,92-14,59$ \\
Apgar $1<7$ & $1,02 \pm 0,49$ & 0,0352 & 2,79 & $1,07-7,25$ \\
\hline
\end{tabular}

OR = ODDS RATIO; IC(95\%) = Intervalo de Confiança; IG = idade gestacional

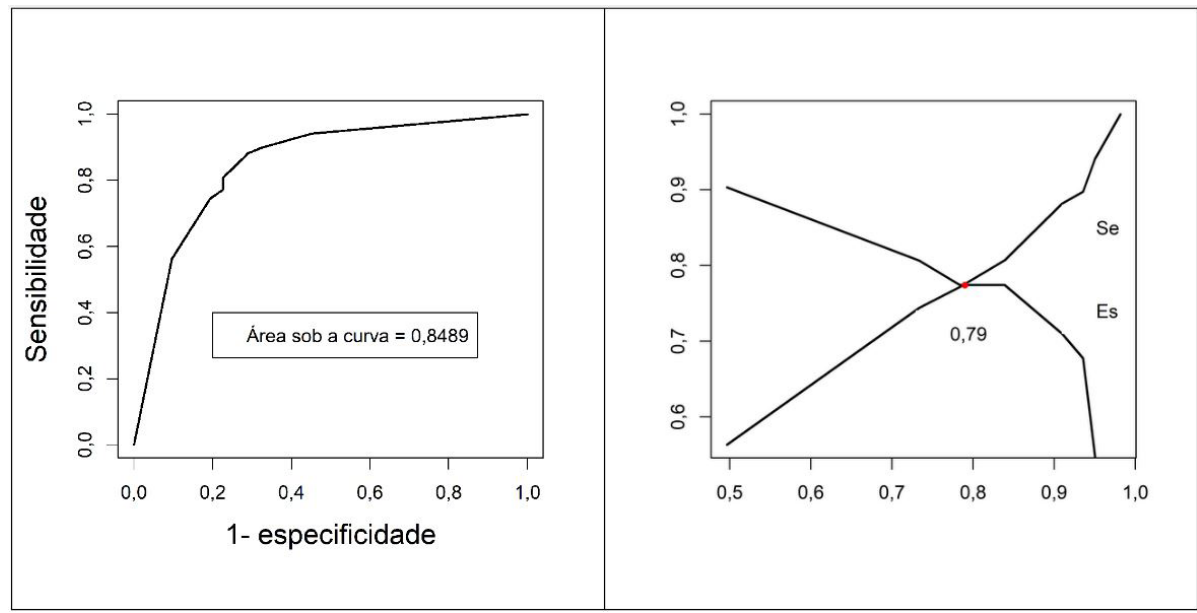

Figura 1. Curva ROC e TG-ROC para Retinopatia da prematuridade (ROP)

medidas de peso ao nascer, idade gestacional e escore de Apgar no primeiro minuto, e este apresentar uma probabilidade maior que 0,79 , então será classificado como recém-nascido com ROP; caso contrário, será classificado como recém-nascido sem a ROP.

\section{DISCUSSÃO}

Neste estudo, a prevalência da ROP foi de $10,81 \%$, percentual abaixo do esperado, se comparado a outros estudos brasileiros, como o de Graziano et al. ${ }^{13}$, com 29,9\%, Bonotto et al. ${ }^{14}$, com $20 \%$, de Tomé et al. ${ }^{1}$, que foi de $44,6 \%$, de Shinsato et al. ${ }^{15}$, com $35,71 \%$ e o estudo de Schumann et al. ${ }^{16}$, em que a ROP chegou a atingir mais da metade da população, com prevalência de $53,14 \%$. Dentre os estudos citados, a grande maioria avalia, para o estudo da ROP, os recém-nascidos com peso menor ou igual a 1.500 gramas e/ou idade gestacional menor ou igual que 32 semanas.

A proporção do sexo masculino e feminino dentre os que apresentaram o diagnóstico de ROP não apontou uma diferença significativa na probabilidade de ter ROP, enquanto que, no estudo de Sola et al. ${ }^{17}$, o sexo masculino e a raça branca foram fatores de risco para retinopatia.

A retinopatia é uma doença que está associada a multifatores; por conseguinte, há vários fatores a serem considerados no estudo, sendo que a idade gestacional, o peso ao nascer e a oxigenoterapia são encontrados com predominância na literatura ${ }^{2,13,18}$. O presente estudo apontou correlação com a ROP pela análise univariada com os fatores: idade gestacional, peso ao nascer, escore Apgar do primeiro e quinto minuto, e dias totais de internação.

Acrescentando, ainda, que, para o presente estudo, a variável oxigenoterapia não apresentou significância estatística, devido, provavelmente, ao fato da falta de informações, pois não foi possível obter nos prontuários a concentração de oxigênio utilizada e nem o tempo em que o recém-nascido esteve sob oxigenoterapia.

Em um estudo recente ${ }^{1}$, o tempo médio de oxigenoterapia foi estatisticamente significante entre o grupo com ROP e o grupo sem ROP, apresentando médias de 31,02 dias e 10,42 dias, respectivamente, evidenciando a associação entre ROP e o uso prolongado de oxigênio. O estudo de Sola et al. ${ }^{17}$ relata a forte relação entre a oxigenoterapia e a ROP, e lembra a necessidade de pesquisas que falem dos níveis suficientes de oxigênio para cada recém-nascido, com o intuito de prevenir os casos mais graves.

Uma das limitações de nosso estudo foi a falta dos registros de variáveis importantes, como a oxigenoterapia, além de os resultados dos exames com diagnósticos da ROP não estarem registrados em formulários específicos do exame realizado pelo retinólogo, dificultando, assim, a obtenção das informações. Observe-se, ainda, que os mesmos, na maioria das vezes, estavam registrados na evolução médica nos prontuários das crianças, 
evidenciando a inexistência de um protocolo único avaliativo das reais condições de saúde física e visual das crianças.

Um dos pontos a salientar neste estudo é a categorização para as variáveis associadas à ROP. Alguns estudos usam como ponto de corte para peso ao nascer menor ou igual que 1.500 gramas e/ou idade gestacional menor ou igual a 32 semanas $^{19,20}$. Neste estudo, houve a preocupação de selecionar os pontos de corte que melhor discriminassem os grupos heterogêneos dentro da população e homogêneos dentro dos grupos, sendo que tais pontos foram validados através do teste da razão de verossimilhança.

O uso do modelo logístico múltiplo em estudos na área de saúde tem, majoritariamente, se restringido a avaliar os fatores associados a um determinado desfecho. Neste estudo, entretanto, foi apresentada a capacidade de predição do modelo pela curva ROC, que mostrou ter capacidade de predição considerável. Assim, recém-nascidos prematuros com probabilidade maior que 0,79 , obtida pelo modelo logístico múltiplo proposto, devem ser avaliados com maior rigor. O modelo logístico múltiplo apontou como grupo de maior risco os recém-nascidos com peso ao nascer menor ou igual que 1.250 gramas e/ou idade gestacional menor ou igual que 30 semanas e/ou escore Apgar no primeiro minuto menor que 7 .

Assim como neste estudo, escore Apgar no primeiro minuto menor que 7 apresentou-se como um fator de risco para a ROP em outros estudos ${ }^{3,14}$. No presente estudo, observou-se que o grupo com ROP apresentou pontuações mais baixas no Apgar comparado ao grupo sem ROP. Além disso, Apgar no primeiro minuto continuou a apresentar significância estatística no modelo múltiplo.

O modelo para a predição quanto ao diagnóstico da ROP pode servir como um teste auxiliar para a prevenção da doença. O grupo de maior risco deve merecer maior atenção dos retinólogos e neonatologistas durante os programas de triagem, destacando que o diagnóstico na fase inicial da doença é fundamental.

Estudar os dados do programa Atenção Humanizada ao Recém-Nascido de Baixo Peso - Método Canguru, sob qualquer ponto de vista, auxilia o profissional de saúde quanto à informação do perfil dos recém-nascidos e isto reflete em melhorias para a comunidade que necessita do atendimento de qualidade dos hospitais.

\section{REFERÊNCIAS}

1. Tomé VAV, Vieira JF, Oliveira LB, Pinto RM, Abdallah VO. Study of retinopathy of prematurity in a university hospital. Arq Bras Oftalmol. 2011;74(4):279-82. PMid:22068856

2. Sousa Costa FA, Gumieiro DEVB, Sfair LC, Oliveira MF, Cardoso AS, Pinto AGT, et al. Retinopatia da Prematuridade: uma revisão. Rev Med Saude Brasilia. 2013;2(1):37-45.

3. Graziano RM, Leone CR. Problemas oftalmológicos mais freqüentes e desenvolvimento visual do pré-termo extremo. J Pediatr. 2005;81(1):95100. http://dx.doi.org/10.2223/1308. PMid:15858668.

4. Zin A, Florêncio T, Fortes JB Fo, Nakanami CR, Gianini N, Graziano RM, et al. Proposta de diretrizes brasileiras do exame e tratamento de retinopatia da prematuridade (ROP). Arq Bras Oftalmol. 2007;70(5):875-83. http://dx.doi.org/10.1590/S0004-27492007000500028. PMid:18157319.

5. Lermann VL, Fortes JB Fo, Procianoy RS. The prevalence of retinopathy of prematurity in very low birth weight newborn infants. J Pediatr. 2006;82(1):27-32. http://dx.doi.org/10.2223/JPED.1433. PMid:16532144.

6. Brasil. Ministério da Saúde. Secretaria de Atenção à Saúde. Departamento de Ações Programáticas Estratégicas. Atenção humanizada ao recém-nascido de baixo peso: Método canguru. 2. ed. Brasília: Ministério da Saúde; 2011.

7. American Academy of Pediatrics. Red reflex examination in infants. Pediatrics. 2002;109(5):980-1. PMid:11986467.

8. Roveri PF, Corrêa DAM, Gonzales HF, Dianin LC, Busatto V. Perfil do bebês atendidos no ambulatório canguru. In: Anais do $9^{\circ}$ Fórum de Extensão e Cultura da UEM; 2011; Maringá. Maringá: UEM; 2011.
9. International Committee for the Classification of Retinopathy of Prematurity. The International Classification of Retinopathy of Prematurity Revisited. Arch Ophthalmol. 2005;123(7):991-9. http://dx.doi.org/10.1001/ archopht.123.7.991. PMid:16009843.

10. Brasil. Secretaria de Atenção à Saúde. Departamento de Ações Programáticas Estratégicas. Coordenação Geral de Saúde da Criança e Aleitamento. Nota técnica $n^{\circ} 11 / 2015$ : diretrizes para a prevenção da retinopatia da prematuridade. Brasília: Secretaria de Atenção à Saúde; 2015.

11. Hosmer DW, Lemeshow S. Applied logistic regression. New York: John Wiley \& Sons; 2000. http://dx.doi.org/10.1002/0471722146.

12. SAS Institute. SAS/STAT(R) user's guide: the GENMOD procedure [Internet]. North Carolina: SAS; 2016 [citado em 2016 mar 28]. Disponível em: http:// support.sas.com/index.html

13. Graziano RM. Retinopatia da prematuridade: contribuição ao estudo da ocorrência e análise dos fatores de risco [tese]. São Paulo: Faculdade de Medicina, Universidade de São Paulo; 1994.

14. Bonotto LB, Moreira ATR, Carvalho DS. Prevalência de retinopatia da prematuridade em prematuros atendidos no período de 1992-1999 em Joinville (SC): avaliação de riscos associados. Arq Bras Oftalmol. 2007;70(1):55-61. http://dx.doi.org/10.1590/S0004-27492007000100011. PMid:17505720.

15. Shinsato RN, Paccola L, Gonçalves WA, Barbosa JC, Martinez FE, Rodrigues MLV, et al. Frequência de retinopatia da prematuridade em recém-nascidos no Hospital das Clínicas da Faculdade de Medicina de Ribeirão Preto da Universidade de São Paulo. Arq Bras Oftalmol. 2010;73(1):60-5. http://dx.doi.org/10.1590/S0004-27492010000100011. PMid:20464116. 
16. Schumann RF, Barbosa ADM, Valete CO. Incidência e gravidade da retinopatia da prematuridade e sua associação com morbidade e tratamentos instituídos no Hospital Universitário Antonio Pedro, entre 2003 a 2005. Arq Bras Oftalmol. 2010;73(1):47-51. http://dx.doi.org/10.1590/S000427492010000100008 . PMid:20464113.

17. Sola A, Chow L, Rogido M. Retinopatía de la prematuridad y oxigenoterapia: una relación cambiante. Ann Pediatr. 2005;62(1):48-63. http://dx.doi. org/10.1157/13070182. PMid:15642242.

18. Motta MMS. Retinopatia da prematuridade limiar em crianças submetidas à terapia com surfactante endotraqueal [tese]. São Paulo: Escola Paulista de Medicina, Universidade Federal de São Paulo; 1997.
19. Fortes JB Fo, Eckert GU, Valiatti FB, Santos PGB, Costa MC, Procianoy RS. The influence of gestational age on the dynamic behavior of other risk factors associated with retinopathy of prematurity (ROP). Graefes Arch Clin Exp Ophthalmol. 2010;248(6):893-900. http://dx.doi.org/10.1007/ s00417-009-1248-6. PMid:20016911.

20. Fortes JB Fo, Eckert GU, Valiatti FB, Costa MC, Bonomo PP, Procianoy RS. Prevalência e fatores de risco para a retinopatia da prematuridade: estudo com 450 pré-termos de muito baixo peso. Rev Bras Oftalmol. 2009;68(1):22-9. http://dx.doi.org/10.1590/S0034-72802009000100005.

Recebido em: Out. 02, 2017 Aprovado em: Mar. 17, 2018 